\title{
Optimism and self-esteem are related to sleep. Results from a large community based sample
}

Sakari Lemola, Ph.D. ${ }^{\text {a, }}$, Katri Räikkönen, Ph.D. ${ }^{\mathrm{b}}$, Veronica Gomez, Ph.D. ${ }^{\mathrm{a}}$, Mathias Allemand, Ph.D. ${ }^{\mathrm{c}}$

${ }^{\text {a }}$ University of Basel, Department of Psychology, Missionsstrasse 62a, 4055 Basel, Switzerland

${ }^{\mathrm{b}}$ University of Helsinki, Faculty of Behavioural Sciences, Siltavuorenpenger 5 A, P.O. Box 9, 00014 University of Helsinki, Finland

${ }^{\mathrm{c}}$ University of Zürich, Department of Psychology, Binzmühlestrasse 14/24, 8050 Zürich, Switzerland

Running head:

Optimism and self-esteem are related to sleep

Author Contact Information:

Sakari Lemola, $\mathrm{PhD}$.

Missionsstrasse 62a

4055 Basel

Switzerland

Tel.: +41612670638

Fax.: +41612670661

Email: Sakari.Lemola@unibas.ch

Conflict of interest: No author indicated any conflicts of interest

\section{Citation:}

Lemola, S., Räikkönen, K., Gomez, V. \& Allemand, M. (in press). Optimism and self-esteem are related to sleep. Results from a large community-based sample. International Journal of Behavioral Medicine. DOI: 10.1007/s12529-012-9272-z. 


\begin{abstract}
Purpose: There is evidence that positive personality characteristics such as optimism and self-esteem are important for health. Less is known about possible determinants of positive personality characteristics. We therefore tested the relationship of optimism and self-esteem with insomnia symptoms and sleep duration.
\end{abstract}

Method: Sleep parameters, optimism, and self-esteem were assessed by self-report in a community-based sample of 1805 adults aged between 30 and 84 years in the United States. Moderation of the relation between sleep and positive characteristics by gender and age as well as potential confounding of the association by depressive disorder was tested.

Results: Individuals with insomnia symptoms scored lower on optimism and self-esteem largely independent of age and sex, and controlling for symptoms of depression and sleep duration. Short sleep duration $(<6 \mathrm{~h})$ was related to lower optimism and self-esteem when compared to individuals sleeping 7-8 hours controlling depressive symptoms. Long sleep duration ( $>9 \mathrm{~h}$ ) was also related to low optimism and self-esteem independent of age and sex. Conclusion: Good and sufficient sleep is associated with positive personality characteristics. This relationship is independent of the association between poor sleep and depression.

Keywords: Insomnia symptoms, sleep duration, optimism, self-esteem, major depression. 


\section{Introduction}

The importance of nocturnal sleep for physical and mental health is widely acknowledged and has been extensively studied. Sleeping less than 7 hours as well as sleeping more than 8 hours is associated with a variety of physical and psychological health problems, such as increased risk of coronary heart disease, hypertension, and depression and anxiety disorders. ${ }^{1-3}$ In addition to short and long sleep duration, insomnia is highly co-morbid with mood and anxiety disorders and longitudinal evidence suggests that insomnia predates the onset of mental illness. ${ }^{4}$

Compared to the large number of studies on negative physical and mental health consequences of poor sleep, knowledge on the relationship of sleep duration and sleep quality with positive individual characteristics, which may promote health and well-being, is scarce. One study showed that individuals who slept on average $7-8$ hours reported more satisfaction with life compared to others who slept less than 6 hours a night. ${ }^{5}$ Moreover, longer sleep duration prospectively predicted higher self-esteem in adolescents, ${ }^{6}$ and adequate sleep duration and short sleep onset latency related to higher optimism in children. ${ }^{7}$ Further, in an experimental study, Haack and Mullington ${ }^{8}$ demonstrated that sleep deprivation resulted in a gradual reduction of self-reported optimism and sociability in young adults, which suggests a causal relation between sleep and positive personality characteristics.

The study of positive personality characteristics such as optimism and self-esteem is important due to their well-known links with subjective well-being and health. ${ }^{9}$ Longitudinal studies indicate that optimism and self-esteem are predictors of better health rather than just consequences. Orth and colleagues ${ }^{10}$ for instance showed that low levels of self-esteem is a vulnerability factor predisposing individuals to depression while there was no evidence for the reverse causality. Another prospective study over a 9 year follow-up period revealed a protective effect of dispositional optimism against all-cause and cardiovascular mortality in old age controlling for the initial health status. ${ }^{11}$ A possible explanation for this effect is that 
more optimistic individuals have more adaptive coping strategies and better health behavior, which may decrease the risk of cardiovascular morbidity.

In the present study, we therefore tested associations of sleep parameters with optimism and self-esteem in a large community based sample of adults aged 30 to 84 years. In particular, we tested relations between insomnia symptoms and positive personality characteristics and examined whether individuals with an average sleep duration (between 7 and 8 hours/day) are more optimistic and have higher self-esteem compared to individuals who sleep less than 7 hours or, conversely, more than 8 hours per day. As it has been documented that poor sleep is comorbid with depressive symptoms and optimism and selfesteem are both inversely related with depressive symptoms, we additionally test whether sleep and positive characteristics are related beyond their shared overlap with depression. Furthermore, since insomnia symptoms may be partly co-morbid with short sleep duration, we additionally tested the relation of insomnia symptoms and sleep duration with positive personality characteristics controlling for the respective other sleep variable. Finally, as sleep parameters undergo age-related changes and show sex specific disparities ${ }^{12}$ moderation of the relationship by participants' age and sex was assessed.

\section{Methods}

\subsection{Participants}

The data for this study come from the MIDUS (Midlife in the U.S.) survey, which was designed to investigate factors contributing to age-related differences and changes in physical and psychological health, and social responsibility. The survey started in 1995 , followed by a reassessment in 2005. MIDUS is a national probability sample, drawn with random-digit dialing procedures, that consists of English-speaking, non-institutionalized adults with at least one telephone per household. The sample was stratified by age and sex, with oversampling of older people and of men. The response rate in the first wave was $71 \%$ with a sample size of $N$ $=3,487$ respondents. Of those participants, 1805 (52\%; 818 males and 987 females) 
completed a self-administered questionnaire in the follow-up containing questions on insomnia symptoms, sleep duration, and positive personality characteristics. Sample retention was selective towards higher participation of whites, females, and married individuals as well as individuals with better health and more education. ${ }^{13}$ Since no information on habitual sleep duration was obtained in the first measurement wave, only data from the second wave were analyzed in the present study. Information on insomnia symptoms was available for $N=1797$, valid information on sleep duration on weekdays and weekends was available for $N=1755$. The average age was 56.9 years $(S D=12.6$, range $=30-84$ years $)$.

\subsection{Measures}

\subsubsection{Insomnia symptoms and sleep duration.}

Insomnia symptoms were measured with three items ("Do you have trouble falling asleep," "Do you wake up during the night and have difficulty going back to sleep," and "Do you wake up too early in the morning and be unable to get back to sleep") using a 5-point scale ranging from never (1) to almost always (5). These three items were summed into a single insomnia symptoms score with a Cronbach's alpha of .81. Sleep duration was assessed by asking participants to indicate how many hours and minutes they generally sleep on weekdays or workdays, and on weekends or non-workdays. For the present analyses, sleep duration on weekdays and weekends was combined by the weighted average.

\subsubsection{Dispositional optimism.}

Dispositional optimism was measured with the Life Orientation Test-Revised (LOT-R ${ }^{14}$ ), a 6item self-report measure assessing generalized expectancies for positive and negative outcomes. Participants were asked to rate the extent to which they agreed with each statement on a 5-point Likert-type scale ranging from strongly agree (1) to strongly disagree (5). The scale was coded such that higher values indicate higher optimism. Cronbach's alpha for the LOT-R in the present sample was .79. Compared to the original version of the LOT-R ${ }^{14}$ the four filler items were omitted. 


\subsubsection{Dispositional self-esteem.}

Dispositional self-esteem was measured with a 7-item version of the Self-Esteem Scale $\left(\mathrm{SES}^{15}\right)$, a widely used scale to assess global attitudes about the self. Participants were asked to indicate their agreement on a 7-point Likert-type scale ranging from strongly agree (1) to strongly disagree (7). The scale was coded such that higher values indicate higher selfesteem. Cronbach's alpha for the SES in the present sample was .76.

\subsubsection{Depression.}

Depression was assessed using the screening version of the World Health Organization's (WHO) “Composite International Diagnostic interview," Version 1.0 (CIDI) ${ }^{16}$, which was administered by phone call. The scale ranged from 0 (no symptoms of depression) to 7 (highest depression), 205 participants (11.4\%) had scores of 4 or more and met criteria for major depressive disorder according to DSM-III-R in the 12 months prior to the survey. In the present study depression scores were used as continuous variable.

\subsection{Statistical analyses.}

To test the relation of optimism and self-esteem with sleep, insomnia symptoms were treated as continuous variable, while sleep duration was dummy coded with 7-8 hours serving as reference category against which further categories of sleeping less than 6 hours, sleeping 6-7 hours, 8-9 hours, and sleeping more than 9 hours were tested. Multiple regression analyses using IBM SPSS 20.0 (IBM Corp. Armonk, NY, USA) were conducted in five steps: first, only age and sex were adjusted; second, models additionally adjusted depression scores; third, models additionally adjusted the respective other sleep parameter (i.e., when sleep duration was the predictor insomnia symptoms were adjusted and vice versa) since insomnia symptoms and short or long sleep duration may be co-morbid conditions; fourth, models included a sleep $\times$ participant's sex interaction term; and finally, the models included a sleep $\times$ participant's age interaction term. The moderated regression analyses were conducted as suggested by Aiken and West. ${ }^{17}$ 


\section{Results}

\subsection{Mean values, sex differences, and correlations of study variables}

Table 1 shows mean values and correlations of the study variables. On average participants reported to sleep 7.14 hours. There was a modest increase of sleep duration with age but no correlations between insomnia symptoms and age was found. Gender comparisons revealed that women had more insomnia symptoms than men $(F(1 / 1795)=34.6, p<0.001)$, while there were no sex differences in sleep duration $(F(1 / 1753)=0.5 ; p=0.83)$. Dispositional optimism, self esteem, and depression scores were correlated with age such that older participants reported higher optimism, self-esteem and lower depression scores. Optimism did not vary by participant's $\operatorname{sex}(F(1 / 1791)=3.2 ; p=0.07)$ but men had higher levels of selfesteem $(F(1 / 1789)=23.1, p<0.001)$ and lower depression scores $(F(1 / 1803)=41.9, p<$ 0.001). Optimism and self-esteem were positively related to each other and negatively with depression scores. Insomnia symptoms and sleep duration were negatively related. (Insert Table 1 about here)

\subsection{Associations between sleep and positive personality characteristics}

Table 2 shows coefficients of multiple regression analyses testing the relationship of optimism and self-esteem with insomnia symptoms and sleep duration. Insomnia symptoms were related to lower levels of optimism and self-esteem. The relations were only modestly attenuated when further controlling depression scores and sleep duration. Interaction terms with age and sex were statistically not significant. Short sleep duration (sleeping less than 6 hours) was related to lower levels of optimism. The relation remained statistically significant when controlling depression or insomnia symptoms although it was considerably attenuated in size when controlling insomnia symptoms. As the short sleep (sleeping less than 6 hours) $\times$ sex interaction term was statistically significant the analysis was repeated separately for men and women adjusting age. This analysis revealed that short sleep was related to lower optimism in both men $(\beta=-.13, t=-2.45, p=0.02)$ and women $(\beta=-.30, t=-6.50, p<$ 
0.001 ) but the relation was considerably stronger for women. Short sleep (sleeping less than 6 hours) was also negatively related to self-esteem adjusting participant age, sex, and depression but was attenuated to insignificance when adjusting insomnia symptoms. Long sleep (sleeping more than 9 hours) was also negatively related to optimism and self-esteem. This relation was not moderated by participant sex or age. Moreover, the relation of long sleep with lower self-esteem held statistically significant when adjusting depression scores or insomnia symptoms.

(Insert Table 2 about here)

\section{Discussion}

Our findings are generally consistent with previous epidemiological and experimental studies showing that less insomnia symptoms and sleep duration of 7-8h are related to higher levels of positive personality characteristics ${ }^{5-8}$. Our findings are further in line with evidence of better mental health among individuals without insomnia symptoms compared to individuals suffering from troubled sleep ${ }^{4}$. Generally, the pattern of relations of sleep measures with optimism and self-esteem was not moderated by age. With regard to participant's sex, we found that women- as compared to men- appear to be more vulnerable for the negative relation of short sleep duration with optimism. This might reflect a generally higher vulnerability among women for negative outcomes related with inadequate sleep duration. For instance, women with short sleep duration are also at higher risk for hypertension ${ }^{3}$, while no such increased risk was found for men. However, more research is needed to replicate and better understand the sex differences we found. Importantly, the relationship between short sleep and lower optimism was independent of possibly co-morbid insomnia symptoms and held also when controlling depression scores.

Consistent with epidemiological studies that show a relationship of long sleep duration with poor physical and mental health ${ }^{1,2}$ we also found evidence for lower levels of optimism 
and self-esteem in individuals who slept longer than 9 hours compared to their counterparts who sleep 7-8 hours.

Our cross-sectional findings do not allow inferences on causality and underlying mechanisms that link sleep and positive personality characteristics. One possible mechanism that may associate sleep with positive personality characteristics is that short sleep duration and poor sleep quality lead to dysregulation of the hypothalamic-pituitary-adrenocortical (HPA) axis involving changes in cortisol secretion ${ }^{18}$, which in turn is known to be adversely related to positive personality characteristics ${ }^{19}$. Equally, it is possible that positive personality characteristics facilitate better sleep by their beneficial influence on physical and mental wellbeing. Finally, also a bi-directional relationship between sleep and personality characteristics is possible involving different causal mechanisms working at the same time.

A strength of the present study is the use of data from a large community based sample covering a broad age range. However, there are also some limitations. First, as already mentioned, our analyses were only cross sectional and do not inform on the direction of the relationship. Second, sample retention of MIDUS II was selective regarding ethnicity, gender, marital status, higher education, and better health, which might have affected the findings. Third, the data are solely based on self-reported sleep duration and insomnia symptoms. It is therefore possible that memory distortions have influenced the report of sleep. Apart form the self-reports of sleep duration and insomnia, also optimism and self-esteem were self-reported. Thus, the relationship between sleep measures, optimism, and self-esteem might partly be due to same-method variance.

\section{Conclusion}

In sum, our findings extend previous knowledge by showing that having less insomnia symptoms is related to higher levels of dispositional optimism and self-esteem. Further, short sleep duration $(<6 \mathrm{~h})$ was related to lower optimism and self-esteem when compared to individuals who sleep 7-8 hours. 


\section{Acknowledgements}

This research was supported by a grant from the MIDUS Pilot Grant Competition, National

Institute on Aging, USA, to M. Allemand. The publication is based on data from the MIDUS Study, funded by the John D. and Catherine T. MacArthur Foundation Research Network on Successful Midlife Development and the National Institute on Aging (P01-AG020166).

\section{References}

1. John U, Meyer C, Rumpf H-J, Hapke U. Relationships of psychiatric disorders with sleep duration in an adult general population sample. J Psychiat Res. 2005;39:577-83.

2. Meisinger C, Heier M, Löwel H, et al. Sleep duration and sleep complaints and risk of myocardial infarction in middle-aged men and women from the general population: the monica/kora augsburg cohort study. Sleep. 2007;30:1121-7.

3. Stranges S, Dorn JM, Cappuccio FP, et al. A population-based study of reduced sleep duration and hypertension: the strongest association may be in premenopausal women. $\mathrm{J}$ Hypertens. 2010;28:896-902.

4. Buysse DJ, Angst J, Gamma A, Ajdacic V, Eich D, Rössler W. Prevalence, Course, and Comorbidity of Insomnia and Depression in Young Adults. Sleep. 2008;31:473-80.

5. National Sleep Foundation (2002). Executive summary of the 2002 Sleep in America Poll. http://www.sleepfoundation.org/sites/default/files/2002SleepInAmericaPoll.pdf Accessed June 7, 2011.

6. Fredriksen K, Rhodes J, Reddy R, Way N. Sleepless in Chicago: Tracking the effects of adolescent sleep loss during the middle school years. Child Dev 2004;75:84-95.

7. Lemola S, Räikkönen K, Scheier MF, et al. Sleep quantity, quality and optimism in children. J Sleep Res. 2011;20:12-20.

8. Haack M, Mullington JM. Sustained sleep restriction reduces emotional and physical wellbeing. Pain. 2005;119:56-64. 
9. Rasmussen HN, Wrosch C, Scheier MF, Carver CS. Self-regulation processes and health: The importance of optimism and goal adjustment. J Pers. 2006;74:1721-1747.

10. Orth U, Robins RW, Roberts BW. Low self-esteem prospectively predicts depression in adolescence and young adulthood. J Pers Soc Psychol. 2008;95: 695-708.

11. Giltay EJ, Geleijnse JM, Zitman FG, Hoekstra T, Schouten EG. Dispositional optimism and all-cause and cardiovascular mortality in a prospective cohort of elderly Dutch men and women. Arch Gen Psychiat. 2004;61:1126-35.

12. Li RHY, Wing YK, Ho SC, Fong SYY. Gender differences in insomnia - a study in the Hong Kong Chinese population. J Psychosom Res. 2002;53:601-9.

13. Radler BT, Ryff CD. Who participates? Accounting for longitudinal retention in the MIDUS National Study of Well-being. J Aging Health. 2010;22:307-31.

14. Scheier MF, Carver CS, Bridges MW. Distinguishing optimism from neuroticism (and trait anxiety, self-mastery, and self-esteem): A re-evaluation of the life orientation test. J Pers Soc Psychol .1994;67:1063-78.

15. Rosenberg M. Society and the adolescent self-image. Princeton, NJ: Princeton University Press, 1965.

16. Kessler RC, Andrews A, Mroczek D, et al. The World Health Organization Composite International Diagnostic Interview Short-Form (CIDI-SF). Int J Meth Psych Res. 1998;7:171-85.

17. Aiken LS, \& West SG. Multiple regression: testing and interpreting interactions. Thousand Oaks, CA: Sage Publications Inc, 1991.

18. Buckley TM, Schatzberg AF. On the Interactions of the Hypothalamic-Adrenal (HPA) Axis and Sleep: Normal HPA Axis Activity and Circadian Rhythm, Exemplary Sleep Disorders. J Clin Endocr Metab. 2005;90:3106-14. 
19. Pruessner JC, Hellhammer DH, Kirschbaum C, et al. Low self-esteem, induced failure and the adrenocortical stress response. Pers Indiv Differ. 1999;27:477-89. 
Table 1 . Mean values and correlations of study variables.

\begin{tabular}{lcccccc}
\hline & $M(s d)$ & 1. & 2. & 3. & 4. & 5. \\
\hline 1. Age (years) & $56.85(12.62)$ & & & & & \\
2. Optimism & $23.05(4.76)$ & $.09^{* * *}$ & & & & \\
3. Self-esteem & $37.49(7.38)$ & $.09^{* * *}$ & $.60^{* * *}$ & & & \\
4. Depression & $0.67(1.80)$ & $-.13^{* * *}$ & $-.28^{* * *}$ & $-.34^{* * *}$ & & \\
5. Insomnia symptoms & $7.54(2.92)$ & .01 & $-.22^{* * *}$ & $-.27^{* * *}$ & $.23^{* * *}$ & \\
6. Sleep duration (h) & $7.14(1.15)$ & $.05^{*}$ & $.11^{* * *}$ & $.07^{* *}$ & $-.09^{* * *}$ & $-.38^{* * *}$ \\
7. Sleep duration (dummy) & & & & & & \\
$\quad<6 \mathrm{~h}(n=197)$ & - & .07 & $-.22^{* * *}$ & $-.16^{* * *}$ & $.15^{* * *}$ & $.50^{* * *}$ \\
$\quad$ 6-7h $(n=399)$ & - & $-.08^{*}$ & $-.07^{*}$ & $-.08^{* *}$ & .02 & $.20^{* * *}$ \\
$\quad 8-9 \mathrm{~h}(n=450)$ & - & $.13^{* * *}$ & -.02 & -.01 & -.02 & $-.08^{*}$ \\
$\quad>9 \mathrm{~h}(n=86)$ & - & $.18^{* * *}$ & -.06 & $-.12^{* *}$ & .04 & .03 \\
\hline
\end{tabular}

Note. $* \mathrm{p}<.05 .{ }^{* *} \mathrm{p}<.01 .{ }^{* * *} \mathrm{p}<.001$.

Sleep duration categories are dummy coded with the category of 7-8h of sleep $(n=623)$ as reference. 
Table 2

Associations of insomnia symptoms and sleep duration with optimism and self-esteem. Standardized regression coefficients.

\section{Optimism as criterion}

Insomnia symptoms

Sleep duration

$7-8 \mathrm{~h}$ (reference) vs.

$<6 \mathrm{~h}$

$6-7 \mathrm{~h}$

$8-9 \mathrm{~h}$

$>9 \mathrm{~h}$

\section{Self-esteem as criterion}

Insomnia symptoms

\begin{tabular}{|c|c|c|c|c|c|c|c|c|c|}
\hline \multicolumn{2}{|c|}{ Adj.1 } & \multicolumn{2}{|c|}{ Adj.2 } & \multicolumn{2}{|c|}{ Adj.3 } & \multicolumn{2}{|c|}{ Sleep $\times$ Sex } & \multicolumn{2}{|c|}{ Sleep $\times$ Age } \\
\hline$\beta$ & $t$ & $\beta$ & $t$ & $\beta$ & $t$ & $\beta$ & $t$ & $\beta$ & $t$ \\
\hline-.22 & $-9.49 * * *$ & -.17 & $-7.31 * * *$ & -.19 & $-7.51 * * *$ & -.02 & -.51 & .0 & .03 \\
\hline
\end{tabular}

Sleep duration

$7-8 \mathrm{~h}$ (reference) vs.

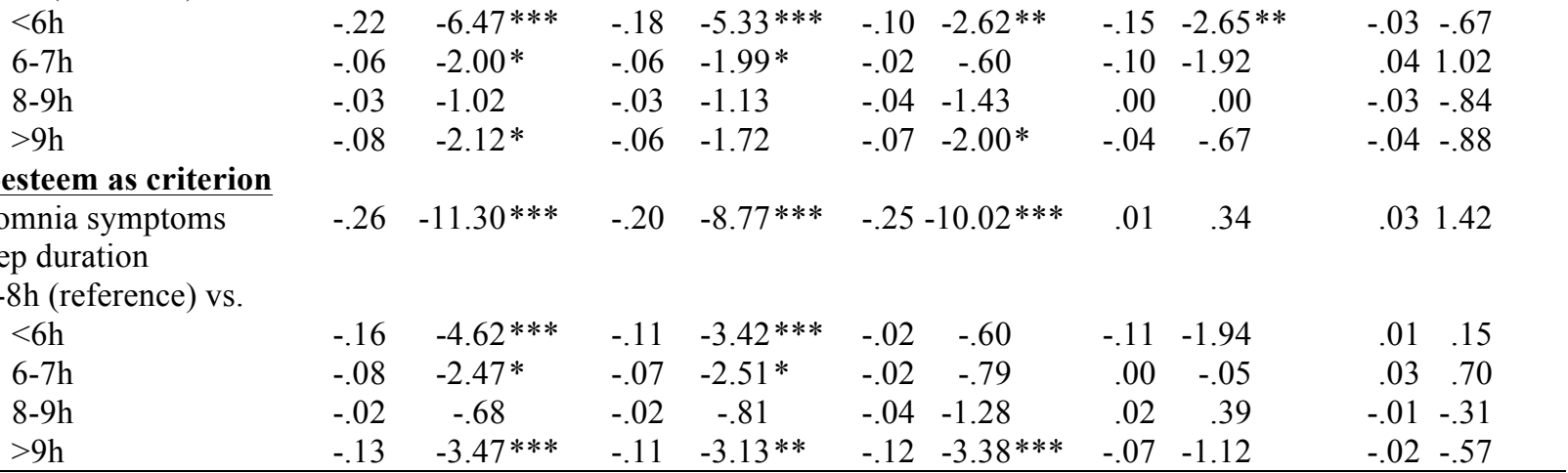

Note. $* \mathrm{p}<.05 . * * \mathrm{p}<.01 . * * * \mathrm{p}<.001$.

Adj.1: Models adjust age and sex.

Adj.2: Models adjust age, sex, and depression scores.

Adj.3: Models adjust age, sex, and the respective other sleep parameter (i.e., models with sleep duration as predictor additionally adjust insomnia symptoms and vice versa).

The models including interaction terms (i.e., insomnia symptoms/sleep duration $\times$ sex or insomnia symptoms/sleep duration $\times$ age) additionally adjust age and sex. 